\title{
ARTICLE
}

\section{Prenatal viral exposure followed by adult stress produces glucose intolerance in a mouse model}

\author{
B. Niklasson • A. Samsioe $\cdot$ M. Blixt $\cdot$ S. Sandler $\cdot$ \\ Å. Sjöholm • E. Lagerquist • Å. Lernmark • W. Klitz
}

Received: 25 February 2006 / Accepted: 2 May 2006 /Published online: 5 July 2006

(C) Springer-Verlag 2006

\begin{abstract}
Aims/hypothesis It has been suggested that the uterine environment may influence metabolic disease occurring later in adult life, and that adult stress may promote disease outcome. Using a mouse model, we tested whether in utero exposure to Ljungan virus (LV) followed by adult exposure to stress produces diabetes. The influence of the timing of viral exposure over the course of pregnancy was also tested.

Materials and methods Pregnant CD-1 mice were exposed i.p. to LV on pregnancy days $4,8,12$ or 17 . Adult male mice from these pregnancies were stressed by being kept in shared cages. Stress only, LV exposure in utero only, and no-stress/no virus exposure groups were also followed.
\end{abstract}

B. Niklasson $(\bowtie)$

Apodemus AB,

Grevgatan 38,

SE-114 53 Stockholm, Sweden

e-mail: Bo.Niklasson@Apodemus.se

B. Niklasson $\cdot$ M. Blixt $\cdot$ S. Sandler

Department of Medical Cell Biology, Uppsala University,

Uppsala, Sweden

A. Samsioe · Å. Sjöholm

Department of Internal Medicine, Karolinska Institutet,

Stockholm South Hospital,

Stockholm, Sweden

E. Lagerquist $\cdot$ A. Lernmark

Department of Medicine, R. H. Williams Laboratory,

University of Washington,

Seattle, WA, USA

W. Klitz

School of Public Health, University of California,

Berkeley and Public Health Institute,

Oakland, CA, USA
Outcome variables included bodyweight, epididymal fat weight, baseline glucose, glucose tolerance tests (60 and $120 \mathrm{~min}$ ) and serum insulin.

Results We demonstrated that male mice developed a type 2-like diabetes, including obesity, as adults if infected during pregnancy with LV. Diabetes at the age of 11 weeks was more severe in mice whose mothers were infected earlier than in those whose mothers were infected later in pregnancy. Only animals infected in utero and kept under stress developed diabetes; infection or stress alone did not cause disease.

Conclusions/interpretation This work demonstrates that a type 2 diabetes-like disease can be virus-induced in a mouse model. Early in utero viral insults can set the stage for disease occurring during adult life, but the final manifestation of diabetes is dependent on the combination of early viral exposure and stress in adult life.

Keywords Diabetes $\cdot$ Fetal programming $\cdot$ Ljungan virus . Mouse model $\cdot$ Stress $\cdot$ Type 2 diabetes

\author{
Abbreviations \\ BW bodyweight \\ GTT glucose tolerance test \\ LV Ljungan virus \\ HPA hypothalamic-pituitary-adrenal
}

\section{Introduction}

Viral infection, the intrauterine environment and stress have all been postulated to play a role in the development of human diabetes [1-3]. The concept of a fetal origin of adult disease is not new, but usually involves fetal 
malnutrition [4]. This concept is based on the Barker hypothesis, originating from observations that the risk for coronary heart disease was correlated with low birthweight and accelerated postnatal growth [5]. Subsequent work has expanded this concept to intrauterine factors other than solely malnutrition, e.g. toxic agents [6] and viral exposure (e.g. cytomegalovirus infection [7]), and endpoints other than cardiovascular disease, e.g. diabetes [8]. As all these intrauterine fetal insults are potentially preventable, it is important to determine how they influence the development and progression of diabetes during adulthood. In an attempt to more realistically mimic the interactive nature of diabetes aetiology, a new rodent model incorporating all three factors is presented here.

We have earlier reported the finding that the incidence of type 1 diabetes correlates with small rodent abundance in Sweden [9]. This and other findings initiated an effort to isolate novel infectious agents from small rodents in an attempt to find an aetiological agent for type 1 diabetes in humans. As a result of these efforts, we isolated a new picornavirus (Ljungan virus [LV]) from bank voles trapped in Sweden $[10,11]$. Moreover, it has been shown that more than one-third of wild-caught bank voles and their offspring develop diabetes when kept under laboratory conditions [12]. This was accompanied by changes in pancreatic islet structure, islet cells becoming immunohistochemically positive for LV and elevated levels of islet autoantibodies [13]. The finding of $\mathrm{LV}$ in the pancreatic islets of the diabetic bank voles raised the general question whether or not LV can participate in the development of diabetes in rodents [13, 14]. To determine if LV is the aetiological agent causing diabetes in wild rodents, a virus-free bank vole colony is necessary. We have failed to maintain LV-free bank voles in captivity for any extended period of time. We therefore turned to LV-free laboratory mice to fulfil Koch's postulates and establish an animal model.

In pilot experiments, we screened a number of mouse strains (CD-1, BALB/c, NMRI and C57BL/6) and infected males at 2 to 3 months of age, but this did not affect glucose tolerance during the follow-up. Next, LV infections were tested following neonatal inoculations and glucose intolerance was observed in some adult animals during the follow-up. The idea of using behavioural stress as an additional factor was also introduced. These initial trials led to the experiments described here, in which LV exposure during pregnancy and social stress in the male offspring are examined for their influence on the development of some of the characteristic indicators of diabetes, including glucose intolerance, bodyweight (BW), fat weight and serum insulin.

\section{Materials and methods}

Animal treatment

Outbred CD-1 mice (Charles River Laboratories, Sulzfeld, Germany) were used. The mice had free access to water and standard laboratory chow (LABFOR R3; Lactamin, Kimstad, Sweden) with an energy content of $3.01 \mathrm{kcal} / \mathrm{g}$. The experimental procedures were approved by the animal ethical committee in Stockholm (N283/05) and were in accordance with international guidelines (NIH publications no. 85-23, revised 1985).

Groups of eight pregnant CD-1 mice were exposed i.p. on days $4,8,12$ or 17 . Mice were infected with $L V$ strain $145 \mathrm{SL}$. The virus stock had been passed six times in suckling mouse brain [10]. Approximately $1,000 \mathrm{ID}_{50}$ were given i.p., while control mice received an i.p. injection of the same volume of saline at the same time. All pregnant females were kept in individual ventilated cages (Techniplast $\mathrm{GmbH}$, Germany). Offspring were weaned from the mother at 3 weeks of age. In the present study, following puberty male mice were kept at either a single animal per cage (the non-stress regimen) or four males in the same cage (the stress regimen). All animals were given glucose $(100 \mathrm{~g} / \mathrm{l})$ in the drinking water as a metabolic stress.

Animal follow-up

At 11 weeks of age, BW was measured and a glucose tolerance test (GTT) was performed. For the GTT, $2 \mathrm{~g}$ glucose $/ \mathrm{kg}$ BW (as a $100 \mathrm{mg} / \mathrm{ml}$ solution) was injected i.p. Blood glucose determinations were performed immediately prior to injection $(0 \mathrm{~min})$ and at 60 and 120 min after injection, and analysed by an automated glucose meter (Precision PCX; Abbott, Stockholm, Sweden). A normal blood glucose level of a mouse is $5-10 \mathrm{mmol} / 1$.

GTT results were summarised by AUC over the three time-points. About 7 to 10 days after the GTT, animals were killed by cervical dislocation and exsanguination via the carotid arteries and blood was collected. The serum insulin level was later measured using a rat insulin high-range ELISA kit (Mercodia AB, Uppsala, Sweden) according to the instructions of the manufacturer. Standardised radioligand-binding assays for autoantibodies against GAD-65 were used to analyse serum samples from mice $[15,16]$.

The weight of the epididymal fat cushion was measured and taken as a marker of the amount of abdominal fat. Standard haematoxylin and eosin staining was carried out on pancreatic sections embedded in paraffin. 


\section{Morphometry}

Upon killing, pancreatic glands were fixed in $4 \%$ buffered paraformaldehyde. The original intention was to evaluate signs of insulitis if a type 1 diabetes-like condition developed. However, since instead a type 2 diabetes-like phenotype evolved, it was decided to assess if there was a change in islet mass. For this, $6-\mu \mathrm{m}$ thick sections were prepared and stained with haematoxylin-eosin. The areas of about three or four whole pancreatic profiles were measured per animal, and next the areas of the islet profiles in the same sections were measured, with the examiner being unaware of the origin of the pancreatic sections. This was performed with a light microscope using a computerised system for morphometry (MOP-Videoplan; Zeiss Svenska, Stockholm, Sweden). For each animal, the islet area in relation to the whole pancreas was calculated. In these experiments, specific immunocytochemistry for insulin was not applied.

\section{Statistics}

Means for the three GTT readings were compared by unpaired $t$-tests. The experiments testing LV/stress and LV timing were evaluated with one-way ANOVA followed by Scheffe multiple-comparisons testing when the overall $F$ statistic was significant. Normality and heterogeneity of variances of distributions were corrected using standard transformations. Because of the small sample sizes and non-parametric nature of the distributions of the raw variables, ANOVA results were confirmed with nonparametric Kruskal-Wallis tests. The five exposures of timing of in utero LV or saline injection were treated as ranked variables and tested for correlation with the four outcome measures of diabetes using the Spearman rank correlation coefficient, rho. Because of the relatively small sample sizes and the violation of assumptions of the ANOVA apparent in the raw variables, in addition to the non-parametric tests and transformations of variables employed, each treatment outcome for the stress and timing hypotheses (Table 1) studies are displayed with box plots to accurately summarise the distribution of variates.

\section{Results}

Three hypotheses regarding the roles of intrauterine LV exposure and adult stress on diabetic outcomes in male CD-1 mice were tested (Table 1). Female CD-1 mice tested under similar experimental conditions in an earlier pilot experiment did not develop diabetes and were therefore not included in this experiment.

These tests are based on the seven distinct experimental treatment groups, which vary on the basis of LV exposure during gestation, timing of LV exposure during gestation and stress as adults (Fig. 1). The first hypothesis was that intrauterine exposure to LV and adult stress leads to glucose intolerance, measured by GTTs and pancreatic morphology. This experiment set the stage for more detailed explorations of type 2-like diabetes in this mouse model. On the basis of the second hypothesis - that both stress and LV are essential for diabetic outcome - we examined the necessity for both factors in producing possible diabetic outcomes, measured as glucose tolerance, body fat, serum insulin and GAD-65 antibodies. Testing of the third hypothesis - that the timing of LV exposure during pregnancy influences later diabetic outcome - was done to find clues as to the nature of the pathogenic effects occurring during gestation.

Mean values of blood glucose were measured at 0,60 and $120 \mathrm{~min}$ of the GTT in CD-1 adult male mice comparing two treatment groups: the untreated control group and the early LV exposure+stress group (Table 1 ). This experiment clearly demonstrated glucose intolerance present in the LV-infected and stressed group (Fig. 2).

Morphological examination of the pancreatic glands upon killing revealed no obvious structural changes of the pancreatic islets, nor any inflammatory lesions. A blinded morphometric analysis on haematoxylin and eosin sections of the percentage islet area in relation to the total pancreatic area was performed in the untreated control group and the early (day-4) LV exposure+stress group. In the untreated control group, the relative islet area was nominally smaller than the early LV exposure+stress group $(0.64 \pm 0.15 \%$, $n=10$ vs $2.0 \pm 0.73 \%, n=9$ ). This putative difference did not, however, quite attain statistical significance $(p=0.07$, unpaired $t$-test).

The relative contributions of adult stress and LV exposure during development were explored by comparing four treatment groups for three outcome variables of diabetes (Table 1). The treatment groups included early LV exposure and stress, LV exposure only, stress only, and a fourth control group exposed to neither LV nor stress.

Glucose tolerance differed among the four treatments by both ANOVA $\left(F_{3,33}=10.01, p<0.001\right)$ and the KruskalWallis test $\left(\chi_{3}^{2}=14.7, p<0.003\right)$. Glucose tolerance, measured as AUC over $120 \mathrm{~min}$, was distributed over a broader range in the early LV exposure and stress-treated compared with that seen in the other three treatments (Fig. 3a). The LV only, stress only and control treatments did not differ from each other. This experiment showed that glucose tolerance in adult male mice was disturbed by LV exposure during gestation, but only in the presence of the additional factor of stress during adulthood.

BW also differed according to experimental treatment (ANOVA, $F_{3,33}=21.03, p<0.001$; Kruskal-Wallis, $\chi_{3}^{2}=15.5$, $p<0.001$ ), and showed a pattern of differences among treatments very similar to that seen for GTT (Fig. 3b): 
Table 1 Experimental design for testing the impact of LV and stress on diabetic outcomes in male CD-1 mice

\begin{tabular}{lll}
\hline Hypothesis & Treatment groups & Outcome variables \\
\hline 1: Is glucose intolerance present in adult male CD-1 mice & 1. In utero LV, adult stress & 1. GTT \\
following in utero LV exposure and adult stress? & 2. No LV (sham injection), no stress & 2. Islet morphology \\
2: Do in utero LV and adult stress both play a role in & 1. In utero LV, adult stress & 1. GTT \\
diabetic outcomes? & 2. Adult stress only & 2. BW \\
& 3. In utero LV only & 3. Body fat (epididymal fat) \\
& 4. No LV, no stress & 4. Serum insulin \\
3: When is the fetus most sensitive to LV exposure? & 1. Day-4 in utero LV, adult stress & 1. GTT \\
& 2. Day-8 in utero LV, adult stress & 2. BW \\
& 3. Day-12 in utero LV, adult stress & 3. Body fat (epididymal fat) \\
& 4. Day-17 in utero LV, adult stress & 4. Serum insulin
\end{tabular}

Treatment groups are used in the analysis of more than one hypothesis

greater magnitude and variance of BW was present in the early LV exposure+stress group. The three other treatments did not differ from one another. Again, as for the GTT results, an influence of $\mathrm{LV}$ on increased $\mathrm{BW}$ was only seen in the context of stress.

Epididymal fat weight was also measured in this same experiment. Following the demonstration of significant group differences by one-way ANOVA $\left(F_{3,33}=8.55\right.$, $p<0.001)$ and the Kruskal-Wallis test $\left(\chi_{3}^{2}=15.5, p<0.002\right)$, multiple-comparison testing showed that epididymal fat was significantly greater in the early LV exposure+stress group compared with the untreated control group (Fig. 3c). For epididymal fat, the LV-only treatment group (early LV exposure and no stress group) was significantly greater than the untreated control group. The stress-only group fell between the LV-only and untreated control group with neither of these differences being significant $(p=0.15$ and $p=0.18$, respectively). These results show that epididymal

\section{Designations}

Day 17

LV day 17

Stress

Day 12

LV day 12

Stress

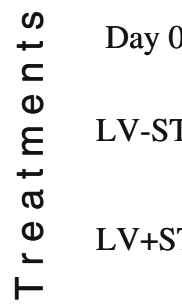

\begin{tabular}{|c|c|}
\hline LV day 12 & Stress \\
\hline LV day 8 & Stress \\
\hline $\mathrm{NaCl}$ day 4 & Stress \\
\hline
\end{tabular}

$\mathrm{LV}+\mathrm{ST}+$ /day $\underline{4 \quad \text { LV day } 4}$

No stress

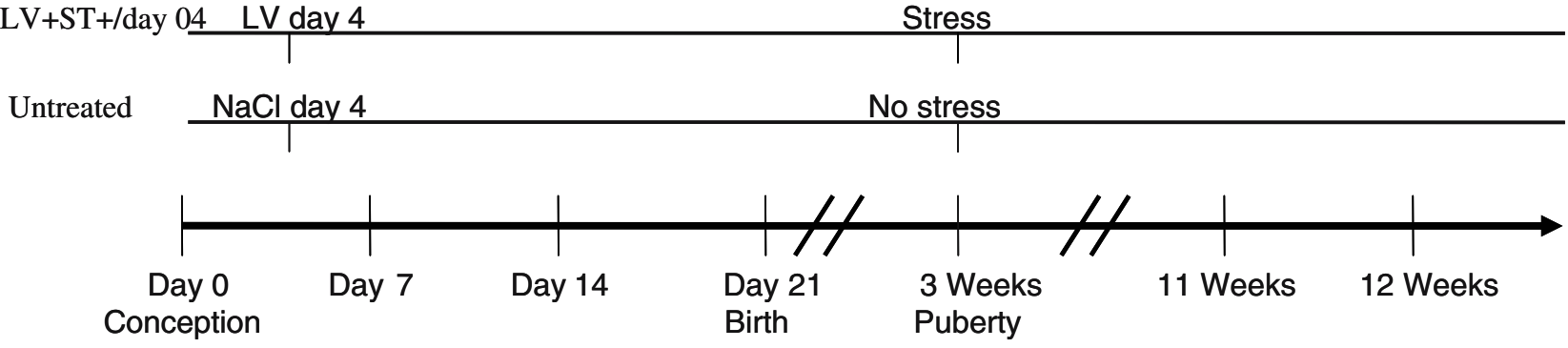

\section{L i f e}

Fig. 1 Treatment groups of male CD-1 mice to test for the influence of in utero LV exposure and adult stress on diabetic symptoms. The stress regimen was initiated at the time of puberty, BW and GTT were measured at 11 weeks, and following killing after 12 weeks of age, serum insulin and epididymal fat measurements were taken. ST Stress 


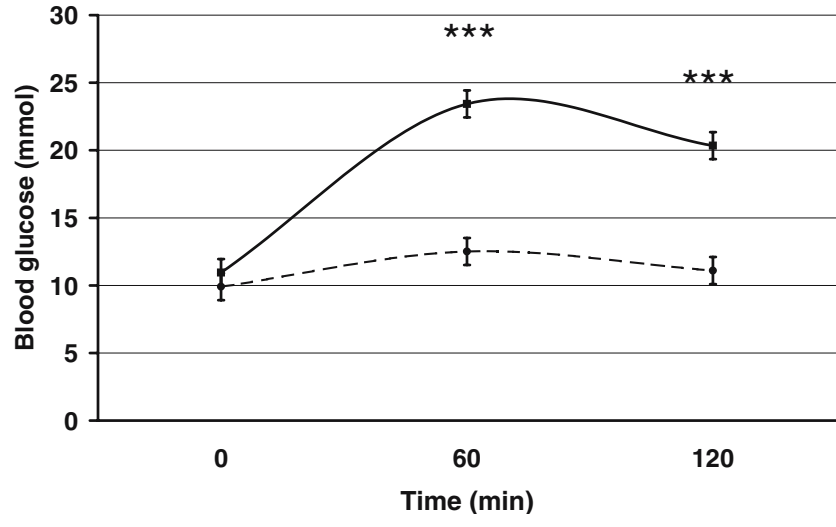

Fig. 2 Glucose tolerance test comparing LV infection followed by adult stress on day 4 (solid line) with controls (dotted line) in CD-1 male mice. A total of nine animals were used for the LV+stress group and eight for the control group. ${ }^{* * *} p<0.001$, unpaired $t$-test

fat was increased in both the early LV exposure+stress group and the early LV exposure and no stress group, compared with the untreated control group.

A few observations of serum insulin were outliers with very high values, making the variances between treatments extremely heterogeneous. After transformation of the raw observations to achieve normality and homogeneity of variances, the treatments did not differ by ANOVA $\left(F_{3,33}=1.80, p=0.17\right)$ or the Kruskal-Wallis test $\left(\chi_{3}^{2}=5.7\right.$, $p=0.12)$.

GAD-65 autoantibodies were tested as an indicator of type 1 diabetes. Sera from all animals collected at the time of killing were tested. All sera from all groups included in this study were negative for GAD-65 autoantibodies.

The third hypothesis (Table 1) concerns the importance of the timing of intrauterine exposure of LV on diabetic outcomes. This experiment examined the impact of LV exposure during pregnancy with five groups of pregnant dams infected i.p. on gestational days 4, 8, 12, 17 and a fifth unexposed group (Fig. 4). Because both LV and stress were demonstrated to be necessary for producing diabeteslike symptoms in the offspring (hypothesis 2), all LV exposure groups were also exposed to the adult-stress regimen. Each of three outcome variables, glucose tolerance, BW and epididymal fat, differed according to the timing of LV exposure during development (Kruskal-Wallis test $\chi_{4}^{2}=17.6, p=0.002 ; \quad \chi_{4}^{2}=25.2, p<0.001 ; \chi_{4}^{2}=16.3$, $p=0.003$, respectively). Furthermore, there was a significant trend for these differences to decline from greatest impact in early exposure to least impact in no exposure for each of the outcome variables: Spearman's rank correlation coefficients were $-0.61,-0.73$ and -0.54 (all at $p<0.001$ ) for GTT, BW and fat, respectively. BW and fat each showed a tendency for an influence of LV gestational exposure all the way to day 17 (Fig. 4). This is supported by multiplecomparison tests following significant one-way ANOVA results for both variables, in which the untreated control group was lower than day-17 LV treatment for BW and lower than for fat. This was not true for GTT, in which neither day-17 nor day-12 treatments differed from the untreated group ( $p=0.40$ and 0.75 , respectively).

The correlation among the diabetic outcome variables is a measure of the tendency for a clustering of the primary metabolic symptoms of type 2 diabetes. For this test, four diabetic indicator variables, GTT, BW, epididymal fat and serum insulin, and all 60 mice from the seven independent treatment groups were used. Positive correlations were present for all six of the comparisons with five reaching statistical significance. The Spearman rank correlation
Fig. 3 The effect of LV infection and stress (combinations of LV or saline administered on pregnancy day 4 and stress or no stress on adult male offspring) on glucose tolerance (a), BW (b) and epididymal fat (c). The box plot for each treatment group indicates the median value, the interquartile range, and the next value greater and less than the interquartile range. Sample sizes for the $\mathrm{LV}+\mathrm{ST}+$, $\mathrm{LV}+\mathrm{ST}-, \mathrm{LV}-\mathrm{ST}+$, and untreated groups were nine, ten, eight and ten, respectively. All significant $p$ values based on multiple-comparison testing between pairs of treatments are shown: ${ }^{\mathrm{a}} p=0.001,{ }^{\mathrm{b}} p=0.015,{ }^{\mathrm{c}} p=0.000$, ${ }^{\mathrm{d}} p=0.000,{ }^{\mathrm{e}} p=0.001,{ }^{\mathrm{f}} p=0.000$ $\mathrm{g}_{p=0.012,}{ }^{\mathrm{h}} p=0.000$
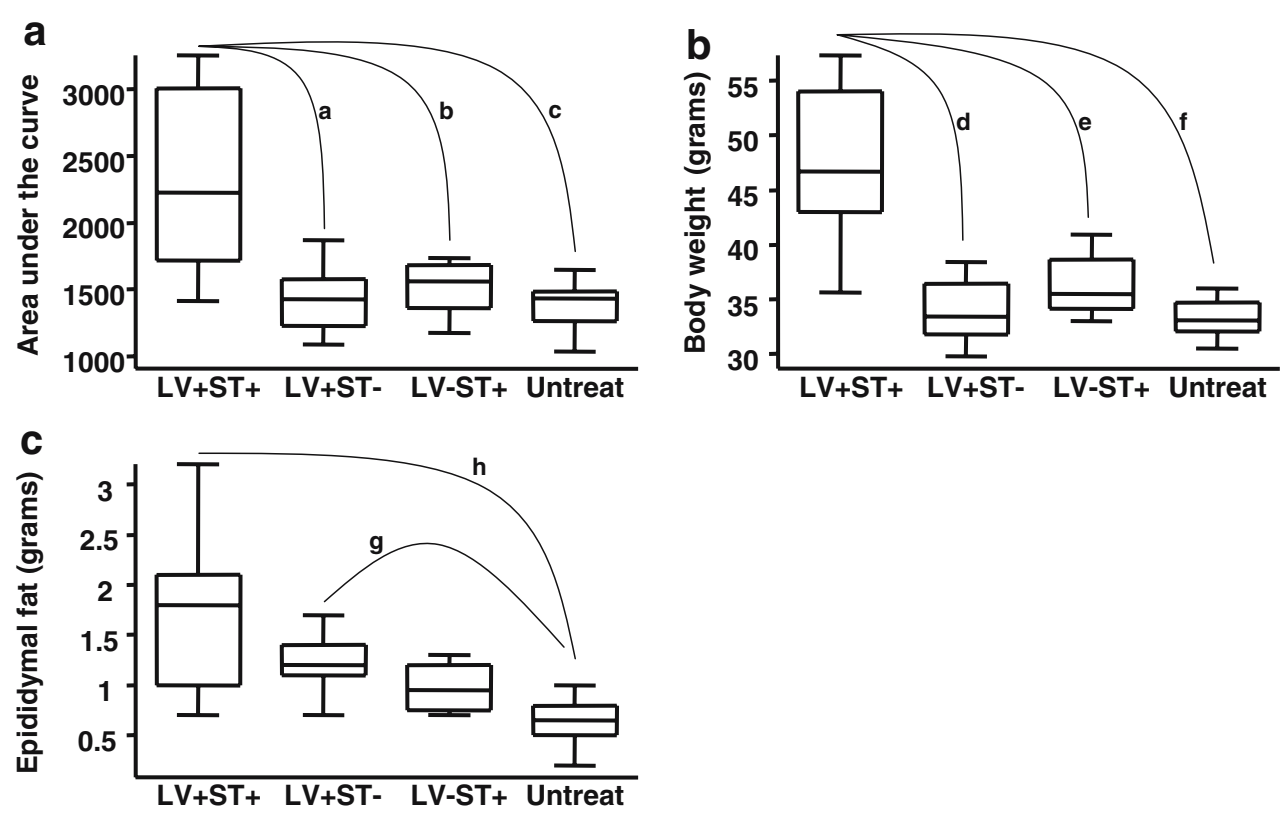
Fig. 4 The effect of the timing of LV infection during gestation on glucose tolerance (a), BW

(b) and epididymal fat (c). The 'untreated' group consisted of animals that were exposed to neither LV nor stress, while the other four groups were all exposed to LV and stress. The box plot for each group indicates the median value, the interquartile range, the next value greater and less than the interquartile range, and two outlier values $(\mathrm{O})$. Sample sizes for day 4 , day 8 , day 12 , day 17 and control groups were nine, eight, eight, seven and ten, respectively. Selected $p$ values from multiple pair-wise comparison tests following ANOVA are shown: ${ }^{\mathrm{a}} p=0.75,{ }^{\mathrm{b}} p=0.40,{ }^{\mathrm{c}} p=0.006$, ${ }_{p}=0.06$,
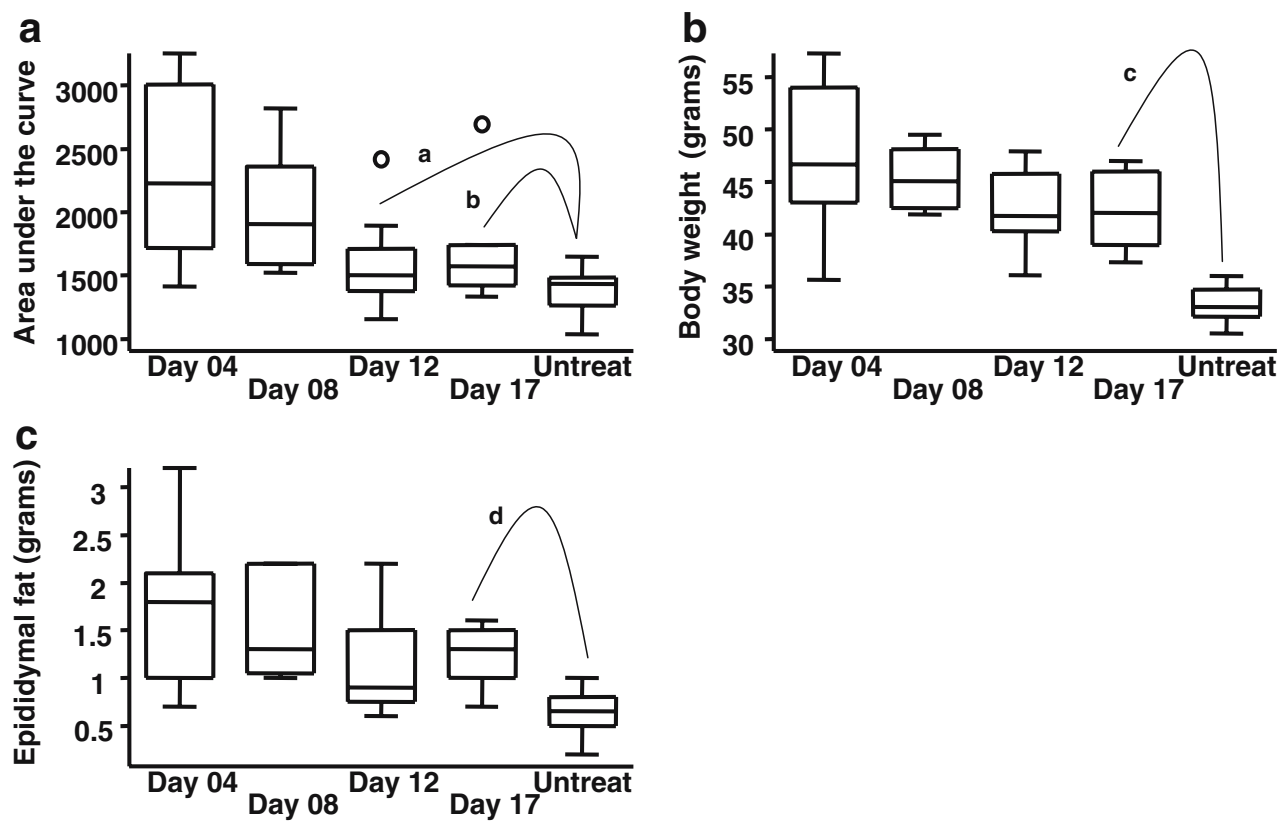

coefficients were $\mathrm{BW} \times \mathrm{GTT}, 0.62$; fat $\times \mathrm{GTT}, 0.52$; insulin $\times$ GTT, 0.13 ; fat $\times$ BW, 0.69; BW $\times$ insulin, 0.29 ; fat $\times$ insulin, 0.55. All values were significant at $p<0.001$, except for insulin $\times$ GTT, which was non-significant, and $\mathrm{BW} \times$ insulin, for which $p<0.05$. The fact that the highest correlation was between fat and BW suggests that BW changes may be a proxy for changes in abdominal fat, reflected here in measurements of epididymal fat. Serum insulin levels are in significantly positive correlation with BW and epididymal fat, but not with GTT. These results point to a coherent assemblage of diabetic characteristics, in which individuals tend to either possess diabetic symptoms or not.

\section{Discussion}

Creating a new rodent model of diabetes, we investigated whether LV infection during the prenatal period might affect the glucose homeostasis in CD-1 mice. Viral exposure did indeed impair glucose tolerance and enhanced other diabetic characteristics in the male offspring of treated dams. It was found that LV infection during pregnancy altered glucose tolerance and led to markedly elevated BW in the adult male offspring. In addition, behavioural stress applied to the adult offspring was found to be an essential factor in the induction of diabetic symptoms. The data also show that the timing of viral exposure over the course of gestation is an additional factor influencing diabetic outcomes: the earlier in pregnancy the viral exposure, the more severe the diabetic signs.

Our results fit the concept of developmental origin (or fetal programming) of adult disease [4], but also expand this model inasmuch as they implicate viral exposure, rather than malnutrition, as the fetal insult. The biological basis for the Barker hypothesis has been suggested to involve developmental plasticity, i.e. a period in utero in which a certain organ is plastic and sensitive to environmental influences [17]. It has been proposed that lifelong programming of the hypothalamic-pituitary-adrenal (HPA) axis in utero is an important mechanism in explaining the onset of chronic degenerative disease in adulthood [18]. However, the relationships are complex and both hyper- and hypocortisolism may arise as a consequence of fetal programming of the HPA axis during intrauterine life. Nonetheless, although not scrutinised in our present work, such a relationship between fetal events and adult-onset diabetes is intriguing, given the role of glucocorticoids and stress in the pathogenesis of type 2 diabetes [19].

The first observation of LV-related diseases was recently made in bank voles, which showed that animals in captivity developed diabetes [13, 14]. Polyuria, polydipsia, glucosuria, elevated blood glucose levels, ketoacidosis and deaths were observed. Lysis of pancreatic islet beta cells and presence of GAD-65, IA-2 and insulin autoantibodies suggested that the bank voles suffered from a type 1 diabetic condition [13]. Bank voles colonised for more than a decade were studied in more detail and it was found that approximately $20 \%$ of the animals developed an abnormal GTT, accompanied by high serum insulin levels and enhanced beta-cell function at basal glucose levels, consistent with type 2 diabetes (Blixt M, Niklasson B and Sandler $\mathrm{S}$, unpublished observations). Moreover, total destruction of beta cells was also found in some of these colonised bank voles, similar to the pathology observed in recently trapped voles [13]. These animals thus seem to go through a type 2 diabetes-like phase with viable beta cells, evidence of 
insulin resistance indicated by abnormal GTTs and high serum insulin levels, where some progress to type 1 diabetes with total destruction of beta cells. Cardio virus is genetically related to LV and is an intensively studied and established diabetes mouse model [20]. It has recently been found that cardio virus can cause both a type 1-like and a type 2-like diabetes in mice [20,21]. Present and previous data therefore suggest that a viral infection can cause both type 1 diabetes and type 2 diabetes. The outcome may depend on the susceptibility of the host. High susceptibility results in type 1 diabetes while a lower susceptibility results in type 2 diabetes. Intermediate susceptibility may show a period of type 2 diabetes-like aberrations in wild voles, where some of the animals develop the end-stage of disease as type 1 diabetes.

Measurements of epididymal fat revealed a suggestion that LV exposure alone may influence fat content (Fig. 3c). This was not, however, carried over to the other outcome variables. For GTT and BW, the combination treatment was essential for diabetic responses (Fig. 3a,b), as the unifactorial treatments revealed no differences from the untreated controls.

A wide range of response was seen for the most diabetogenic treatment $(\mathrm{LV}+$ stress $)$ across all variables measured, showing that individual mice respond differently with some attaining the full complement of diabetic indicators and others avoiding diabetes symptoms altogether. This might in part reflect genetic differences among the outbred CD-1 strain used in these studies. The genetic diversity underlying their outbred status probably contributes to the variable response. Genic heterozygosity, which comes with genetic diversity, tends to reduce developmental noise, which may be an important source of statistical noise in experiments employing congenic strains. The outbred state represents a more accurate model of variation in the outside world, among both native rodents and humans. It is worth pointing out that differences in food intake among individuals, unfortunately not measured in our experiments, may have also influenced outcomes.

The coherence of three primary symptoms of type 2 diabetes, GTT, body fat and serum insulin levels, demonstrated through significant correlations among them, strengthens the supposition that a type 2 diabetes-like disease was created in some individuals. Demonstrations of diabetogenic effects were not, in general, present for serum insulin levels. The rather small sample sizes employed in this study were only capable of detecting substantial influences, so it is possible that differences in insulin output were too small to see. In addition, extreme outlier values of serum insulin seen in some treatments made the small sample sizes especially difficult to manage statistically.

It is well recognised that the developmental program of an organism is most vulnerable in its earlier stages. The experiments on the timing of $\mathrm{LV} /$ stress exposure during development (Fig. 4) established the fact that exposure to LV earlier in pregnancy leads to more severe symptoms. This suggests that the most vulnerable developmental stage in this model occurred no later than day 4. For glucose tolerance the susceptible period may decline more sharply after day 8, while effects on BW and fat may extend throughout pregnancy, but these conclusions may be near the limits of statistical power for the experiments.

In two groups of mice we measured the pancreatic islet tissue in relation to the exocrine tissue by morphometry. There was an evident trend $(p=0.07)$ that the LV-infected mice had an expansion of the islet mass. This suggests that during the development of glucose intolerance an increase in islet mass may occur as compensation for disturbed glucose homeostasis. Alternatively, the LV infection may be acting on the growth of the islet tissue itself. While serum insulin levels were not significantly correlated with islet area, it was found that the extreme outlier value, which occurred in serum insulin and in islet area, was in the same (early LV exposure+stress group) individual.

We found that distinctive environmental effects occurring at opposite ends of the lifespan synergistically combine to promote the diabetic state. This work establishes a mouse model for studying a complex, yet realistic, model of human diabetes. We found that $\mathrm{LV}$ infection of mice during pregnancy can induce glucose intolerance in stressed adult male mice. The features of the condition seen in CD-1 male mice bear evident similarity to that seen in human type 2 diabetes.

Acknowledgements We are grateful for the excellent technical assistance of I.-B. Hallgren. This work was supported in part by National Institutes of Health grant NIDDK064365, the Barndiabetes fonden, the Swedish Research Council (72X-8273), the Swedish Diabetes Association, the Novo Nordisk Fund and the Family Ernfors Fund.

Duality of interest The authors have no dualities of interest.

\section{References}

1. Jun HS, Yoon JW (2003) A new look at viruses in type 1 diabetes. Diabetes Metab Res Rev 19:8-31

2. Barker DJ, Hales CN, Fall CH, Osmond C, Phipps K, Clark PM (1993) Type 2 (non-insulin-dependent) diabetes mellitus, hypertension and hyperlipidaemia (syndrome $\mathrm{X}$ ): relation to reduced fetal growth. Diabetologia 36:62-67

3. Golden SH, Williams JE, Ford DE et al (2004) Depressive symptoms and the risk of type 2 diabetes: Atherosclerosis Risk in Communities Study. Diabetes Care 27:429-435

4. Barker DJ (2005) The developmental origins of insulin resistance. Horm Res 64(Suppl 3):2-7

5. Barker DJ, Gluckman PD, Godfrey KM, Harding JE, Owens JA, Robinson JS (1993) Fetal nutrition and cardiovascular disease in adult life. Lancet 341:938-941 
6. Lau C, Rogers JM (2004) Embryonic and fetal programming of physiological disorders in adulthood. Birth Defects Res C Embryo Today $72: 300-312$

7. Brakefield PM, Gems D, Cowen T et al (2005) What are the effects of maternal and pre-adult environments on ageing in humans, and are there lessons from animal models? Mech Ageing Dev 126:431-438

8. McKeigue PM, Lithell HO, Leon DA (1998) Glucose tolerance and resistance to insulin-stimulated glucose uptake in men aged 70 years in relation to size at birth. Diabetologia 41:1133-1138

9. Niklasson B, Hörnfeldt B, Lundman B (1998) Could myocarditis, insulin-dependent diabetes mellitus, and Guillain-Barré syndrome be caused by one or more infectious agents carried by rodents? Emerg Infect Dis 4:187-193

10. Niklasson B, Kinnunen L, Hörnfeldt B et al (1999) A new picornavirus isolated from bank voles (Clethrionomys glareolus). Virology 255:86-93

11. Johansson S, Niklasson B, Maizel J, Gorbalenya AE, Lindberg AM (2002) Molecular analysis of three Ljungan virus isolates reveals a new, close-to-root lineage of the Picornaviridae with a cluster of two unrelated 2A proteins. J Virol 76:8920-8930

12. Schoenecker B, Heller KE, Freimanis T (2000) Development of stereotypies and polydipsia in wild caught bank voles (Clethrionomys glareolus) and their laboratory-bred offspring. Is polydipsia a symptom of diabetes mellitus? Appl Anim Behav Sci 68:349-357

13. Niklasson B, Heller KE, Schonecker B et al (2003) Development of type 1 diabetes in wild bank voles associated with islet autoantibodies and the novel Ljungan virus. Int J Exp Diabesity Res 4:35-44

14. Niklasson B, Hörnfeldt B, Nyholm E, Niedrig et al (2003) Type 1 diabetes in Swedish bank voles (Clethrionomys glareolus): signs of disease in both colonized and wild cyclic populations at peak density. Ann N Y Acad Sci 1005:170-175

15. Mire-Sluis AR, Das RG, Lernmark $\AA$ (2000) The world health organization international collaborative study for islet cell antibodies. Diabetologia 43:1282-1292

16. Grubin CE, Daniels T, Toivola B et al (1994) A novel radioligand binding assay to determine diagnostic accuracy of isoformspecific glutamic acid decarboxylase antibodies in childhood IDDM. Diabetologia 37:344-350

17. Ellison PT (2005) Evolutionary perspectives on the fetal origins hypothesis. Am J Hum Biol 17:113-118

18. Kajantie E, Phillips DI, Andersson S et al (2002) Size at birth, gestational age and cortisol secretion in adult life: foetal programming of both hyper- and hypocortisolism? Clin Endocrinol (Oxf) 57:635-641

19. Buren J, Eriksson JW (2005) Is insulin resistance caused by defects in insulin's target cells or by a stressed mind? Diabetes Metab Res Rev 21:487-494

20. Jun HS, Yoon JW (2001) The role of viruses in type I diabetes: two distinct cellular and molecular pathogenic mechanisms of virus-induced diabetes in animals. Diabetologia 44:271-285

21. Utsugi T, Kanda T, Tajima Y et al (1992) A new animal model of non-insulin-dependent diabetes mellitus induced by the NDK25 variant of encephalomyocarditis virus. Diabetes Res 20:109-119 